\title{
Silodosin as a predisposing factor of Intraoperative Floppy Iris Syndrome (IFIS): an observational propensity score-matching cohort study
}

\section{Chrysanthos D. Christou}

Aristotle University of Thessaloniki: Aristoteleio Panepistemio Thessalonikes

Marianna Kourouklidou

Aristotle University of Thessaloniki: Aristoteleio Panepistemio Thessalonikes

Asimina Mataftsi

Aristotle University of Thessaloniki: Aristoteleio Panepistemio Thessalonikes

Eirini Oustoglou

Aristotle University of Thessaloniki: Aristoteleio Panepistemio Thessalonikes

Nikolaos Ziakas

Aristotle University of Thessaloniki: Aristoteleio Panepistemio Thessalonikes

Argyrios Tzamalis ( $\nabla$ argyriostzamalis@yahoo.com )

Royal Liverpool University Hospital https://orcid.org/0000-0002-3172-8542

\section{Research Article}

Keywords: Silodosin, Intraoperative Floppy Iris Syndrome, IFIS, a1-blockers

Posted Date: April 30th, 2021

DOI: https://doi.org/10.21203/rs.3.rs-439878/v1

License: (9) (i) This work is licensed under a Creative Commons Attribution 4.0 International License.

Read Full License

Version of Record: A version of this preprint was published at International Ophthalmology on October 5th, 2021. See the published version at https://doi.org/10.1007/s10792-021-02054-y. 


\section{Abstract}

Purpose: To evaluate the correlation between silodosin and Intraoperative Floppy Iris Syndrome (IFIS) and compare it with other a1- adrenergic receptor antagonists (a1-ARAs) and other factors predisposing to IFIS.

Methods: From the cases who underwent phacoemulsification between 2014 and 2020, we identified all patients who, during their preoperative assessment, reported an a1-ARAs intake (exposed group). These patients were matched utilizing a propensity score matching analysis, with an otherwise homogenous group of patients (control group), based on demographics and systemic/ocular comorbidities.

Results: 350 patients were included in each group. In the exposed group, 177 (50.6\%) patients were exposed to tamsulosin, $105(30 \%)$ to alfuzosin, $43(12.2 \%)$ to silodosin. Regarding IFIS, it was observed in $21.5 \%$ of patients on tamsulosin (38/177), $11.4 \%$ on alfuzosin (12/105), $37.2 \%$ on silodosin (16/43), and $3.4 \%$ in the controlled group (12/350). In a multiple regression model analysis, the only two factors that were significantly associated with IFIS development were silodosin and tamsulosin yielding an adjusted odds ratio of 8.471 (95\%Cl: 4.005-17.920), and 3.803 (95\%Cl: $2.231-6.485)$, respectively.

Conclusion: Silodosin has been demonstrated as a predisposing factor, strongly correlated with IFIS development. These results should increase awareness to cataract surgeons, to carefully assess their patients preoperatively for exposure to silodosin, and employ the appropriate prophylactic measures to ameliorate the impact of silodosin intake on the surgical outcome.

\section{Key Messages}

\section{What is known:}

- a1- adrenergic receptor antagonists (a1-ARAs), and especially tamsulosin, are considered to be the main predisposing factors for the development of Intraoperative Floppy Iris Syndrome (IFIS). However, there is very limited data regarding the correlation between the intake of silodosin, a newest uroselective a1-ARA, and IFIS

\section{New information:}

- In an observational cohort study with propensity score-matching, silodosin, demonstrated strong correlation to IFIS development and should be classified among the main risk factors for the appearance of floppy iris.

- Silodosin intake seems to have a higher odds ratio than any other factor predisposing to IFIS, and therefore, it should be included in the preoperative assessment of cataract surgery in order to minimize the detrimental effects of IFIS by employing the appropriate prophylactic measures.

\section{Introduction}


Intraoperative Floppy Iris Syndrome (IFIS) was defined in 2005 by Chang and Campbell as the occurrence of the following triad of signs during phacoemulsification: an iris which billows and ripples in response to phaco fluidics, a pupil that progressively contracts and poorly responds to mydriatic agents, and finally, an iris stroma which tends to prolapse through the incisions ${ }^{1}$. IFIS is classified based on the above signs' presence as mild, moderate, or severe (presence of one, two, or all three signs respectively) ${ }^{2}$. In the original report, IFIS was utterly attributed to the intake of tamsulosin, a uroselective a1- adrenergic receptor antagonist (a1-ARA), which is commonly prescripted to men as treatment of benign prostate hyperplasia (BPH) ${ }^{1}$. IFIS mostly became apparent after the revised clinical guidelines of BPH's treatment, which replaced surgical intervention with a1-ARAs as the first-line treatment ${ }^{3}$. However, IFIS has subsequently been correlated with a plethora of predisposing factors, including demographics (male gender and advanced age), arterial hypertension and antihypertensive drugs, a poorly dilated pupil, and other medications such as other a1-ARAs (besides tamsulosin), finasteride, antipsychotics, and benzodiazepines ${ }^{4-10}$. Nevertheless, up to date, tamsulosin remains the predisposing factor that is most strongly correlated with IFIS occurrence.

This strong correlation between tamsulosin and IFIS could be attributed to tamsulosin's strong affinity for the $a 1_{A}$ sub-type of the adrenergic receptor $\left(a 1_{A}-A R\right)$, which is significantly more robust than other a1ARAs such as alfuzosin, doxazosin, prazosin, and terazosin ${ }^{11-14}$. The $a 1_{A}$-AR regulates the tone of the musculus dilatator pupillae ${ }^{15}$. Therefore, the inhibition of these receptors interferes with iris's dilation, while their long-term use leads to the alteration of iris' anatomy, specifically the dilator muscle's atrophy that is irreversible and independent of a1-ARAs cessation ${ }^{16-18}$.

Silodosin is the newest member of the a1-ARAs family, granted approval by the Food and Drug Administration in $2008{ }^{19}$. Silodosin is a uroselective a1-ARA with a high affinity for the a ${ }_{A}{ }^{-A R}$.

Therefore, it is fair to believe that silodosin could significantly be correlated to IFIS. However, the literature investigating the correlation between the intake of silodosin and IFIS development is limited ${ }^{20-23}$. Our study aims to evaluate the correlation between silodosin and IFIS. To the best of our knowledge, it represents the first attempt to estimate the power of this correlation, compare it with other a1-ARAs, while adjusting for other predisposing factors linked to IFIS.

\section{Methods And Materials}

\subsection{Study Design and Patient Selection}

We conducted a retrospective observational cohort study in accordance with the STROCSS 2019 Guidelines and the tenets of the Declaration of Helsinki after approval of the Institutional Review Board with a waiver of consent ${ }^{24}$. The study pool consisted of all patients who underwent phacoemulsification at a tertiary ophthalmology department during a 6-year period (2014-2020). Initially, we identified all patients, who during their preoperative assessment (7 days preoperatively), reported an a1-ARAs intake (exposed group). We then conducted a propensity score matching analysis using variables (potential risk 
factors) included in our standard preoperative assessment to match the patients with a1-ARAs intake with an otherwise homogenous group of patients (control group) ${ }^{25}$. Specifically, patients were matched based on: gender, age, diabetes mellitus, arterial hypertension, preceded vitrectomy, corneal opacities, shallow anterior chamber $(<2.5 \mathrm{~mm})$, posterior polar cataract, white/brunescent cataract, pseudoexfoliation, iridodonesis/phacodonesis, glaucoma, deep-set eye, monocularity, and finally, poor compliance of the patient.

\subsection{Data Collection and Definition of Outcomes}

All study data were retrieved after careful assessment of the patients' medical files and the respective electronic operating records. We throroughly reviewed all folders regarding the exposure to risk factors predisposing to IFIS. Specifically, for patients on a1-ARAs, we recorded which particular drug they were administrated, including finasteride. For all patients, we also identified which patients were exposed to benzodiazepines, neuromodulating drugs (including antidepressants, antipsychotics, cholinergic drugs, and dopaminergic drugs), and antihypertensive drugs (angiotensin II receptor inhibitors, b-blockers). We considered a positive exposure, any drug intake, independently of the duration of intake or drug cessation. Finally, for each patient, we recorded the IFIS status. Positive status was considered the occurrence of any of the three signs of IFIS. IFIS was also classified as mild, moderate, and severe (based on the incidence of one, two, or three signs, respectively).

\subsection{Surgical Procedure}

On surgery's day, our protocol included a pre-operative dilation with tropicamide $0.5 \%$, phenylephrine hydrochloride $2.5 \%$, and cyclopentolate $1 \%$ eyedrops. Regarding anesthesia, topical anesthesia was achieved with proparacaine hydrochloride $0.5 \%$, while intracameral anesthesia was achieved with an ophthalmic viscosurgical device containing $1 \%$ sodium hyaluronate and $1 \%$ lidocaine (Visthesia, Zeiss Meditec, Germany). The operation was performed using a $2.4 \mathrm{~mm}$ clear corneal incision and two side ports. For all cases, the same gravity-fluids torsional phacoemulsification device was used (Infiniti Vision System, Alcon Laboratories, Inc.).

\subsection{Statistical Analysis}

Statistical analysis was conducted using SPSS software (Version 26.0, IBM $\left.{ }^{\circledR}\right)$. The confidence interval (Cl) was set at $95 \%$, and the level of statistical significance at $p<0.05$. Categorical variables were described using frequencies and percentages while continuous variables using mean \pm standard deviation. Categorical variables were compared using the Pearson chi-square test, with posthoc pairwise comparisons, where appropriate. For continuous variables, Kolmogorov - Smirnov was used as a normality test. Non-normally distributed continuous variables were compared using the Mann-Whitney U test or Kruskal-Wallis $\mathrm{H}$ test with posthoc pairwise comparisons, as appropriate. Finally, a multiple regression model was used to estimate each predisposing factor's adjusted odds ratio in developing IFIS.

\section{Results}




\subsection{Study Population and Baseline Characteristics}

We identified 376 patients exposed to a1-ARAs at the time of operation. Following a propensity score case-matching analysis, 26 patients remained unmatched, while 350 patients (exposed group) were matched with 350 patients in our cataract database based on the variables of our standard preoperative assessment. Therefore, 700 patients were included, 692 males (98.9\%) and eight females (1.1\%), with a mean age of $75.33 \pm 6.96$ years. Table 1 summarizes the differences in the distribution of the baseline characteristics between the exposed and control group, following the case-matching by propensity scores. Notably, no statistically significant differences in the baseline characteristics were observed between the two groups.

\subsection{Exposure to a1-ARAs and IFIS occurrence}

In the exposed group, 177 (50.6\%) patients were exposed to tamsulosin, 105 (30\%) to alfuzosin, 43 $(12.2 \%)$ to silodosin, $3(0.9 \%)$ to doxazosin, $1(0.3 \%)$ to terazosin, and finally, $45(12.86 \%)$ patients were exposed to finasteride. Table 2 demonstrates the IFIS rates of patients on silodosin, tamsulosin, and alfuzosin, as well as the mean preoperative dilated pupil diameter and the rate of utilization of preoperative prophylactic intracameral epinephrine in each sub-group compared to the control group. Notably, the preoperative dilated pupil was significantly smaller in the silodosin and tamsulosin groups than in the alfuzosin and controlled groups ( $p=0.01$ and $p<0.001$ respectively). The use of prophylactic intracameral epinephrine did not differ significantly among the three a1-ARA groups, while predictably, it was significantly higher compared to the control group. Interestingly, IFIS rates of the silodosin subgroup were significantly higher compared to the control group and the alfuzosin group $(p<0.001)$.

None of the patients on doxazosin presented signs of IFIS, while the patient on terazosin presented a mild IFIS (iris billowing). Four patients were exposed both to tamsulosin and alfuzosin, and one was exposed both to tamsulosin and silodosin. None of these five patients presented IFIS signs. Finally, of the 45 patients who were exposed to finasteride, 11 (24.4\%) showed IFIS signs, $3(6.7 \%)$ mild, 5 (11.1\%) moderate, and $3(6.7 \%)$ severe IFIS.

\subsection{Multiple Regression Model}

A multiple regression model analysis was conducted to estimate the OR of each a1-ARA in correlation with IFIS development. Other predisposing factors were included in the model to reach an adjusted estimation of the OR. Specifically, we included the intake of finasteride, benzodiazepines, neuromodulators, and antihypertensive drugs. Antihypertensives were furtherly divided into angiotensin II receptor inhibitors, b-blockers, or other antihypertensives (including diuretics, calcium channel blockers, and angiotensin-converting enzyme inhibitors). The intake of doxazosin and terazosin was not included in the model since very few patients were exposed to these a1-ARAs to conduct any meaningful analysis. Table 3 presents the adjusted ORs of each predisposing factor in the model along with $95 \%$ confidence intervals $(\mathrm{Cl})$. Notably, the only two factors that were significantly associated with IFIS development were silodosin and tamsulosin $(p<0.001)$. 


\section{Discussion}

In this study, we evaluated the correlation between silodosin intake and IFIS development. We managed to quantify this correlation by estimating an odds ratio while adjusting for covariates reported as factors predisposing to IFIS development. Notably, the control group was homogenous to the exposed group regarding demographics, systematic comorbidities, and ocular comorbidities. Our study demonstrated silodosin as a factor strongly predisposing to IFIS development.

The studies thas have already investigated the correlation between silodosin intake and IFIS are limited. Specifically, some case reports have identified silodosin as the predisposing factor of IFIS, with the most recent one reporting a bilateral IFIS occurrence ${ }^{20-22}$. In addition, a recent retrospective cohort study, with 19 patients exposed to silodosin, reported an IFIS occurrence rate in this sub-group of patients of $79 \%$ $(15 / 19)$, which was higher than the rate of IFIS reported in their tamsulosin sub-group of patients $(63 \%$, $10 / 16)^{23}$. In our study, the rate of IFIS development in the silodosin sub-group was also found higher than the rate of IFIS in the tamsulosin sub-group (37.2\% vs. $21.5 \%)$.

To this day, tamsulosin remains the predisposing factor that is most strongly correlated to IFIS occurrence. Univariate analyses and multiple regression models report unadjusted odds ratios for IFIS occurrence when exposed to tamsulosin between 10.67 and $206.5^{6,26,27}$ and adjusted odds ratios between 5.78 and 4058 4,6,26,28. For this reason, a joint statement by the American Society of Cataract and Refractive Surgery and the American Academy of Ophthalmology advocates either to initiate tamsulosin following cataract surgery or to use a non-selective a1-ARA for BPHs treatment in phakic patients ${ }^{29}$.

Silodosin is prescribed for the treatment of symptomatic BPH and lower urinary tract symptoms (LUTS). When compared to tamsulosin, in a recent meta-analysis, based on 13 studies including 2129 randomized participants, both drugs were found equally effective in the management of symptomatic $\mathrm{BPH}$ and LUTS ${ }^{30}$. Along with the prolongation of life expectancy, a larger population of patients on these a1-ARAs will unevitably require cataract surgery in the near future. When silodosin is prescribed to phacik patients, it is highly recommended that the patient's ophthalmologist should be accordingly informed.

IFIS, especially unanticipated, is correlated with significant intraoperative complications ${ }^{1,8,31,32}$. Fortunately, when patients are appropriately assessed preoperatively to adequately stratify the surgical risk, and the necessary prophylactic measures are employed for high-risk patients, complication rates return to their baseline ${ }^{33}$. Therefore, we recommend that all physicians and medical personel being involved in the preoperative evaluation of cataract patients should record the intake of a1-ARAs, and particularly the intake of tamsulosin, and silodosin.

The surgical risk is not linear. This statement is implemented particularly in IFIS. The vast majority of IFIS cases are correlated with a handful of already identified risk factors. Therefore, the cornerstone in addressing IFIS is first to be familiar with these predisposing factors, then assess them properly 
preoperatively, and finally, employ the necessary prophylactic measures to ameliorate their impact on the surgical outcome ${ }^{34}$.

Our study has several limitations. First, its retrospective nature could have introduced information bias in our study. In addition, several patients who were identified as high-risk patients due to their exposure to one or more predisposing factors received intracameral epinephrine preoperatively. Therefore, our analysis probably has underestimated the actual risk associated with exposure to predisposing factors. Finally, despite our best efforts to adjust for covariates, unknown cause/effect relations could have impacted our results.

On the other hand the large sample size and the utilization of propensity scores case-matching significantly strengthens our results. To the best of our knowledge, our study is the first in the literature that has linked silodosin with IFIS, managing to quantify the power of the correlation between silodosin and IFIS, by estimating an odds ratio, while adjusting for other risk factors by using two homogenous groups of patiens achieved by propensity scores case-matching.

In conclusion, silodosin, a uroselective a1-ARA, has been demonstrated in this study as a predisposing factor, strongly correlated with IFIS development. These results should increase awareness to cataract surgeons, to carefully assess their patients preoperatively for exposure to silodosin, and employ the appropriate prophylactic measures to ameliorate the impact of silodosin intake to the surgical outcome.

\section{Declarations}

No proprietary interest in any of the products mentioned in the study. No conflict of interest. No financial grants or funds were received in support of the study.

The contents of this manuscript have not been copyrighted or published previously and will not be submitted elsewhere while the publication process is active. There are no directly related manuscripts or abstracts, published or unpublished, by any authors of this paper.

\section{References}

1. Chang DF, Campbell JR. Intraoperative floppy iris syndrome associated with tamsulosin. J Cataract Refract Surg. 2005;31(4):664-673. doi:10.1016/j.jcrs.2005.02.027

2. Chang DF, Campbell JR, Colin J, Schweitzer C. Prospective masked comparison of intraoperative floppy iris syndrome severity with tamsulosin versus alfuzosin. Ophthalmology. 2014;121(4):829834. doi:10.1016/j.ophtha.2013.10.031

3. Lunacek A, Mohamad Al-Ali B, Radmayr C, et al. Ten years of intraoperative floppy iris syndrome in the era of a-blockers. Cent Eur J Urol. 2018;71(1):98-104. doi:10.5173/ceju.2017.1234

4. Chatziralli IP, Peponis V, Parikakis E, Maniatea A, Patsea E, Mitropoulos P. Risk factors for intraoperative floppy iris syndrome: A prospective study. Eye. 2016;30(8):1039-1044. 
doi:10.1038/eye.2016.122

5. Issa SA, Dagres E. Intraoperative floppy-iris syndrome and finasteride intake. J Cataract Refract Surg. 2007;33(12):2142-2143. doi:10.1016/j.jcrs.2007.07.025

6. Kaczmarek IA, Prost ME, Wasyluk J. Clinical risk factors associated with intraoperative floppy iris syndrome: a prospective study. Int Ophthalmol. 2019;39(3):541-549. doi:10.1007/s10792-018-0840-3

7. Tzamalis A, Malyugin B, Ziakas N, Tsinopoulos I. Angiotensin receptor inhibitors as main predisposing factor for intraoperative floppy iris syndrome in women. $J$ Cataract Refract Surg. 2019;45(5):696-697. doi:10.1016/j.jcrs.2019.01.034

8. Tzamalis A, Matsou A, Dermenoudi M, Brazitikos P, Tsinopoulos I. The role of sex in intraoperative floppy-iris syndrome. J Cataract Refract Surg. 2019;45(1):41-47. doi:10.1016/j.jcrs.2018.08.029

9. Chen AA, Kelly JP, Bhandari A, Wu MC. Pharmacologic prophylaxis and risk factors for intraoperative floppy-iris syndrome in phacoemulsification performed by resident physicians. J Cataract Refract Surg. 2010;36(6):898-905. doi:10.1016/j.jcrs.2009.12.039

10. Christou CD, Tsinopoulos I, Ziakas N, Tzamalis A. Intraoperative floppy iris syndrome: Updated perspectives. Clin Ophthalmol. 2020;14:463-471. doi:10.2147/OPTH.S221094

11. Wilde MI, Fitton A, Sorkin EM. Terazosin. A review of its pharmacodynamic and pharmacokinetic properties, and therapeutic potential in benign prostatic hyperplasia. Drugs Aging. 1993;3(3):258-277. doi:10.2165/00002512-199303030-00007

12. Fulton B, Wagstaff AJ, Sorkin EM. Doxazosin. An update of its clinical pharmacology and therapeutic applications in hypertension and benign prostatic hyperplasia. Drugs. 1995;49(2):295320. doi:10.2165/00003495-199549020-00011

13. Wilde MI, Fitton A, McTavish D. Alfuzosin. A review of its pharmacodynamic and pharmacokinetic properties, and therapeutic potential in benign prostatic hyperplasia. Drugs. 1993;45(3):410-429. doi:10.2165/00003495-199345030-00008

14. Schwinn DA, Roehrborn CG. Alpha1-adrenoceptor subtypes and lower urinary tract symptoms. Int J Urol Off J Japanese Urol Assoc. 2008;15(3):193-199. doi:10.1111/j.1442-2042.2007.01956.x

15. Schwinn DA, Michelotti GA. alpha1-adrenergic receptors in the lower urinary tract and vascular bed: potential role for the alpha1d subtype in filling symptoms and effects of ageing on vascular expression. BJU Int. 2000;85 Suppl 2:6-11. doi:10.1046/j.1464-410x.2000.00061.x

16. Zaman F, Bach C, Junaid I, et al. The floppy iris syndrome - what urologists and ophthalmologists need to know. Curr Urol. 2012;6(1):1-7. doi:10.1159/000338861

17. Goseki T, Ishikawa H, Ogasawara S, et al. Effects of tamsulosin and silodosin on isolated albino and pigmented rabbit iris dilators: possible mechanism of intraoperative floppy-iris syndrome. $J$ Cataract Refract Surg. 2012;38(9):1643-1649. doi:10.1016/j.jcrs.2012.05.025

18. Nuzzi R, Arnoffi P, Tridico F. Best Prophylactic Strategy in Groups at Risk of Intraoperative Floppy Iris Syndrome Development: Comparison Between Atropine Instillation and Adrenaline Intracameral Injection. Open Ophthalmol J. 2018;12(1):34-40. doi:10.2174/1874364101812010034 
19. Drug Approval Package: RAPAFLO (Silodosin) NDA \#022206. Accessed December 11, 2020. https://www.accessdata.fda.gov/drugsatfda_docs/nda/2008/022206s000TOC.cfm

20. Ipekci T, Akin Y, Hoscan B, Tunckiran A. Intraoperative floppy iris syndrome associated with silodosin. Acta Ophthalmol. 2015;93(4):e306. doi:10.1111/aos.12549

21. Chatterjee S, Agrawal D. Silodosin-associated intraoperative floppy iris syndrome. Indian J Ophthalmol. 2017;65(6):538-539. doi:10.4103/ijo.IJO_704_16

22. Ozcura F, Irgat SG. Bilateral Intraoperative Floppy Iris Syndrome Associated with Silodosin Intake. Eurasian J Med. 2020;52(1):100-102. doi:10.5152/eurasianjmed.2019.19017

23. Terauchi Y, Horiguchi H, Shiba T. The Pharmacological Mydriatic Pupil-To-Limbal Diameter Ratio as an Intuitive Predictor for the Risk of Intraoperative Floppy Iris Syndrome. J Ophthalmol. 2018;2018:18. doi:10.1155/2018/2837934

24. Agha R, Abdall-Razak A, Crossley E, Dowlut N, losifidis C, Mathew G. STROCSS 2019 Guideline: Strengthening the reporting of cohort studies in surgery. Int J Surg. 2019;72:156-165. doi:10.1016/j.ijsu.2019.11.002

25. Thoemmes F. Propensity score matching in SPSS. arXiv. 2012;1201.

26. Neff KD, Sandoval HP, Fernández de Castro LE, Nowacki AS, Vroman DT, Solomon KD. Factors Associated with Intraoperative Floppy Iris Syndrome. Ophthalmology. 2009;116(4):658-663. doi:10.1016/j.ophtha.2008.12.026

27. Keklikci U, Isen K, Unlu K, Celik Y, Karahan M. Incidence, clinical findings and management of intraoperative floppy iris syndrome associated with tamsulosin. Acta Ophthalmol. 2009;87(3):306309. doi:10.1111/j.1755-3768.2008.01246.x

28. Chatziralli IP, Sergentanis TN, Papazisis L, Moschos MM. Risk factors for intraoperative floppy iris syndrome: A retrospective study. Acta Ophthalmol. 2012;90(2):152-153. doi:10.1111/j.17553768.2011.02132.x

29. IFIS associated with systemic alpha 1-antagonists. Accessed November 1, 2020. https://www.eyeworld.org/article-intraoperative-floppy-iris-syndrome-ifis--associated-with-systemicalpha-1-antagonists

30. Jung JH, Kim J, MacDonald R, Reddy B, Kim MH, Dahm P. Silodosin for the treatment of lower urinary tract symptoms in men with benign prostatic hyperplasia. Cochrane database Syst Rev. 2017;11(11):CD012615. doi:10.1002/14651858.CD012615.pub2

31. Muqit MMK, Menage MJ. Intraoperative floppy iris syndrome. Ophthalmology. 2006;113(10):18851886; author reply 1886. doi:10.1016/j.ophtha.2006.05.012

32. Lim L-A, Frost A. Iris tears secondary to intraoperative floppy-iris syndrome associated with tamsulosin. J Cataract Refract Surg. 2006;32(10):1777. doi:10.1016/j.jcrs.2006.05.021

33. Chang DF, Osher RH, Wang L, Koch DD. Prospective Multicenter Evaluation of Cataract Surgery in Patients Taking Tamsulosin (Flomax). Ophthalmology. 2007;114(5):957-964. doi:10.1016/j.ophtha.2007.01.011 
34. Tzamalis A, Christou CD, Tsinopoulos I, Ziakas N. Risk factors of floppy iris syndrome: current insights. Expert Rev Ophthalmol 2021. Ahead of Print. doi: 10.1080/17469899.2021.1877539

\section{Tables}

Table 1. Comparison of demographics, systemic and ocular comorbidities between the exposed and the control group

\begin{tabular}{|llll|}
\hline Variables & Exposed Group (n=350) & Control Group (n=350) & p-value \\
\hline Demographics & & & \\
\hline Age & $75.30 \pm 6.40$ & $75.35 \pm 7.48$ & $0.60 \ddagger$ \\
\hline Male Gender & $346(98.9 \%)$ & $346(98.9 \%)$ & $1.00 \dagger$ \\
\hline Systemic Comorbidities & & & \\
\hline Diabetes Mellitus & $101(28.9 \%)$ & $98(28.0 \%)$ & $0.80 \dagger$ \\
\hline Hypertension & $258(73.9 \%)$ & $236(67.4 \%)$ & $0.06 \dagger$ \\
\hline Occular Comorbidities & & & $0.78 \dagger$ \\
\hline Preceeded Vitrectomy & $4(1.1 \%)$ & $5(1.4 \%)$ & $0.36 \dagger$ \\
\hline Corneal Opacities & $12(3.4 \%)$ & $8(2.3 \%)$ & $0.34 \dagger$ \\
\hline Shallow Anterior Chamber & $24(6.9 \%)$ & $18(5.1 \%)$ & $1.00 \dagger$ \\
\hline Posterior Polar Cataract & $2(0.6 \%)$ & $2(0.6 \%)$ & $0.66 \dagger$ \\
\hline White/brunescent cataract & $23(6.6 \%)$ & $26(7.4 \%)$ & $0.27 \dagger$ \\
\hline Pseudoexfoliation & $41(11.7 \%)$ & $32(9.1 \%)$ & $0.31 \dagger$ \\
\hline Phacodonesis/iridosonesis & $6(1.7 \%)$ & $3(0.9 \%)$ & $0.46 \dagger$ \\
\hline Glaucoma/Ocular Hypertension & $39(11.1 \%)$ & $33(9.4 \%)$ & $0.81 \dagger$ \\
\hline Deep-Set Eye & $36(10.3 \%)$ & $38(10.9 \%)$ & $0.63 \dagger$ \\
\hline Monocularity & $19(5.4 \%)$ & $22(6.3 \%)$ & $(4.0 \%)$ \\
\hline Poor Compliance & $10(2.9 \%)$ & & \\
\hline
\end{tabular}

‡: Mann-Whitney U test, †: Pearson Chi-square test

Table 2. IFIS occurrence in each of a1-ARA sub-groups 


\begin{tabular}{|c|c|c|c|c|c|}
\hline & $\begin{array}{l}\text { Silodosin } \\
(n=43)\end{array}$ & $\begin{array}{l}\text { Tamsulosin } \\
(n=177)\end{array}$ & $\begin{array}{l}\text { Alfuzosin } \\
(n=105)\end{array}$ & $\begin{array}{l}\text { Control } \\
\text { group } \\
(n=350)\end{array}$ & $p$ values \\
\hline $\begin{array}{l}\text { Preoperative } \\
\text { Dilated Pupil }\end{array}$ & $6.42 \pm 0.99$ & $6.84 \pm 0.93$ & $7.21 \pm 1.06$ & $7.28 \pm 0.94$ & $\begin{array}{l}p<0.001 \neq, S-T: p=0.75, S-A: \\
p=0.01 \text {, S-C: } p<0.001, T-A: \\
p=0.11 \text {, T-C: } p=0.001, \text { A-C: } \\
p=1.00\end{array}$ \\
\hline $\begin{array}{l}\text { Intracameral } \\
\text { Epinephrine }\end{array}$ & 7 (16.3\%) & 29 (16.5\%) & 10 (9.5\%) & $6(1.7 \%)$ & $\begin{array}{l}p<0.001+, S-T: p=0.95 \text {, S-A: } \\
p=0.18, \text { S-C: } p<0.001 \text {, T-A: } \\
p=0.09, \text { T-C: } p<0.001 \text {, A-C: } \\
p<0.001\end{array}$ \\
\hline \multirow{5}{*}{$\begin{array}{l}\text { IFIS } \\
\text { Mild } \\
\text { Moderate } \\
\text { Severe }\end{array}$} & $\begin{array}{l}16 \\
(37.2 \%)\end{array}$ & 38 (21.5\%) & $\begin{array}{l}12 \\
(11.4 \%)\end{array}$ & $12(3.4 \%)$ & \multirow{5}{*}{$\begin{array}{l}p<0.001+, S-T: p=0.31, S-A \\
p<0.001 \text {, S-C: } p<0.001 \text {, T-A: } \\
p=0.04 \text {, T-C: } p<0.001, A-C: \\
p=0.001\end{array}$} \\
\hline & \multirow{2}{*}{5 (11.6\%) } & 9 (5.1\%) & \multirow{2}{*}{$7(6.7 \%)$} & 2 (0.6\%) & \\
\hline & & $12(6.8 \%)$ & & $4(1.1 \%)$ & \\
\hline & 4 (9.3\%) & 17 (9.6\%) & $2(1.9 \%)$ & $6(1.7 \%)$ & \\
\hline & 7 (16.3\%) & & 3 (2.9\%) & & \\
\hline
\end{tabular}

‡: Kruskal-Wallis $\mathrm{H}$ test with posthoc pairwise comparisons, + : Pearson Chi-square test with posthoc pairwise comparisons, S: Silodosin, T: Tamsulosin, A: Alfuzosin, C: Control Group

Table 3. Multiple regression model for the estimation of adjusted OR of factors predisposing to IFIS.

\begin{tabular}{|llll|}
\hline & Adjusted Odds Ratio & $95 \% \mathrm{Cl}$ & p-value \\
\hline Silodosine intake & 8.471 & $4.005-17.920$ & $<0.001$ \\
\hline Tamsulosin intake & 3.803 & $2.231-6.485$ & $<0.001$ \\
\hline Alfuzosin intrake & 1.762 & $0.863-3.601$ & 0.12 \\
\hline Finasteride intake & 2.112 & $0.987-4.521$ & 0.05 \\
\hline Benzodiazepine intake & 0.791 & $0.340-1.838$ & 0.585 \\
\hline Neuromodulator intake & 1.285 & $0.597-2.765$ & 0.522 \\
\hline Angiotensin II receptor inhibitor intake & 0.816 & $0.488-1.365$ & 0.439 \\
\hline B-blockers intake & 0.771 & $0.454-1.309$ & 0.335 \\
\hline Other antihypertensives intake & 1.268 & $0.765-2.102$ & 0.358 \\
\hline
\end{tabular}

Cl: Confidence Intervals 